CORRESPONDENCE

\title{
Is nature truly healing itself? Spontaneous remissions in Paroxysmal Nocturnal Hemoglobinuria
}

(c) The Author(s) 2021

\author{
Blood Cancer Journal (2021)11:187; https://doi.org/ \\ 10.1038/s41408-021-00582-5
}

\section{Dear Editor,}

Paroxysmal nocturnal hemoglobinuria $(\mathrm{PNH})$ is considered to be curable only through the means of allogeneic stem cell transplantation (SCT) [1]. One of the many fascinating and scientifically instructive aspects of the pathogenesis of this disease is the unique possibility of its spontaneous remission with disappearance of $\mathrm{PNH}$ cell populations and abatement of clinical symptoms, a rare event which has always captivated the research community [2-4]. Due to the orphan nature of the condition, no clinical predictors have been identified so far as harbingers of this phenomenon estimated to occur in up to $15 \%$ (3-15\% according to the different series) of PNH patients between 10 and 20 years from disease onset $[3,5]$. In a classical scenario, exhaustion of the PNH clone (theory of "finite life span") may be associated with reappearance of aplastic anemia (AA), in which the emergence of a PNH clone reflects a semi-maladaptive attempt of recovery [3]. Consequently, one could stipulate that a retraction of the $\mathrm{PNH}$ clone would have to be associated with a compensatory re-establishment of normal hematopoiesis should normal blood counts be maintained [6]. The latter hypothesis is supported by anecdotal cases of PNH following autologous SCT (ASCT), in which PNH expansion would be favored by either postASCT stem cell pool contraction or immune system derangements, with subsequent extinction of the $\mathrm{PNH}$ cell populations upon cessation of these supporting conditions [6, 7]. Environmental changes underlying $\mathrm{PNH}$ clone expansion/contraction may also provide support for the theory of the "neutral drift" which, by applying in silico models of Markovian stochastic dynamics, posits the absence of a selective advantage of PIGA mutant clones $[8,9]$. Furthermore, the recent insights into the AA/PNH pathobiology shed light onto molecular underpinnings of polyclonal vs. oligoclonal hematopoiesis and their dynamics as to the possible acquisition over time of clonal hematopoiesis of indeterminate potential (CHIP) $[10,11]$. Nevertheless, literature data on genetics of PNH spontaneous remission are scarce with to date only one case molecularly characterized by means of modern technologies (e.g., whole-exome sequencing) [2]. Herein, through the application of multiple nextgeneration sequencing (NGS) panels interrogating genes involved in $\mathrm{PNH}$ clonal evolution, we attempted to better discern the genomic mechanisms of its spontaneous remission.

In total, our study cohort included 92 patients with a PNH granulocyte clone size $>20 \%$ and lactate dehydrogenase $>$ x2.5 upper limit of normal with or without evidence of bone marrow failure (see also Supplemental Material) $[12,13]$. Flow cytometry studies for determination of PNH granulocyte/red blood cell (RBCs) size and type (incomplete loss of CD59 i.e., type II vs. complete loss i.e., type III) were performed as previously described $[12,13]$. Deep sequencing of PIGA gene was performed on whole blood DNA using multi-amplicon NGS with primers covering all coding exons [14]. NGS of 62 myeloid genes (Table S1) and HLA class I/II loci along with a newly developed in-house bioinformatic pipeline (Fig. S1) were used for the detection of somatic clonal events (see also Supplemental Material) $[15,16]$.

Among 92 patients with a diagnosis of $\mathrm{PNH}$ (M:F ratio 0.88, median age 38 , range $9-84$ ) $41 \%$ were classified as primary $\mathrm{PNH}(\mathrm{pPNH}), 49 \%$ were secondary to $\mathrm{AA}(\mathrm{sPNH})$ and $10 \%$ were classified as $\mathrm{AA} / \mathrm{PNH}$ overlap syndrome. Overall, patients were clinically followed-up for a median time of 68 months (range, 2-339). Median granulocyte clone size was $73 \%$ (range, 22-99) with the majority of cases (80\%) being classified as having a type III dominant RBCs clone while $20 \%$ having a type II. Within this cohort, a total of 3 patients experienced spontaneous remission at a median time of 20 years (range, 1-22) from PNH diagnosis (Fig. 1).

UPN1 was a 69-year-old male diagnosed with pPNH at the age of 46 after an episode of deep vein thrombosis. He had been managed with prednisone, transfusions and anticoagulation because of recurrent thrombotic episodes. Once available, he was started on eculizumab and later continued on ravulizumab. His initial flow cytometry study revealed the presence of a type III RBCs clone of $40 \%$ and a granulocyte clone of $89 \%$. After 9 years of anticomplement therapy, the patient's clone started a slow decrease and the most recent flow cytometric study revealed a granulocyte clone of $0.02 \%$. Molecular analysis performed at the time of eculizumab start showed a PNH-dominant mutational configuration by adjusted variant allelic frequency (VAF) with PIGA deletion (p.94_95del; adjusted VAF 29\%) and a BCOR nonsense (p.Y1446X; VAF 13\%). No HLA class $\mathrm{I} / \mathrm{II}$ mutations were found in two longitudinal samples collected 1 year before and after eculizumab start. However, at the last sequencing performed after the complete disappearance of the PNH cell population, the patient developed ASXL1 (p.E635Rfs*; VAF 26\%) and ZRSR2 (p.E120Gfs*; adjusted VAF 21\%) mutations with a codominant configuration along with retraction of the previous PIGA lesion. No decrease in blood counts was noted.

UPN2 was a 58-year-old male initially diagnosed with severe AA at the age of 48 and treated with ATG + cyclosporine. At that time, he had a co-existing PNH granulocyte clone of $28 \%$ (AA/PNH overlap syndrome). During the year following immunosuppressive treatment, his $\mathrm{PNH}$ clone rapidly dropped to $1 \%$ and since then has been consistently below $1 \%$ (last $0.1 \%$ ). Therefore, the patient has never received anticomplement therapy. At the time of $\mathrm{PNH}$ clone retraction, no HLA class I/II or myeloid driver mutations were found and PIGA mutations were not detectable. However, longitudinal molecular studies performed after disappearance of PNH cell population revealed the acquisition of ASXL1 p.Q512X mutation at an initial VAF of $23 \%$, which doubled (45\%) at last follow-up 5 years later while normal counts persisted.

UPN3 was a 59-year-old lady diagnosed with pPNH at the age of 30 . She had a granulocyte clone as high as $43 \%$ with a type II RBCs clone of $17 \%$ and a typical PIGA splice site c.981 $+1 \mathrm{G}>\mathrm{A}$ mutation (adjusted VAF 15\%). She was initially treated with transfusions and 
UPN 1

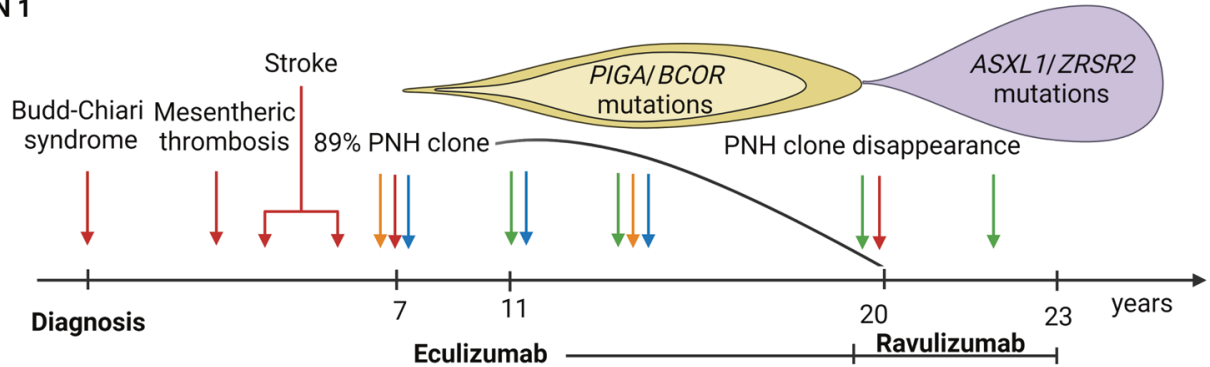

UPN 2

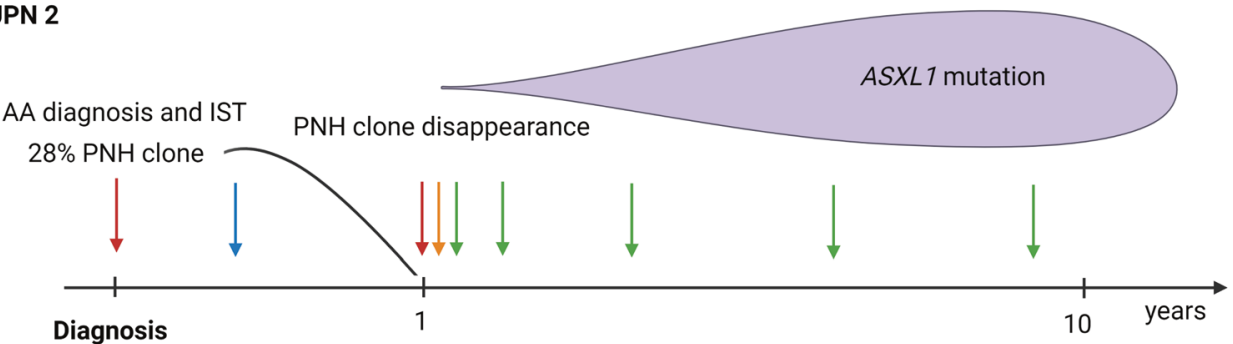

UPN 3

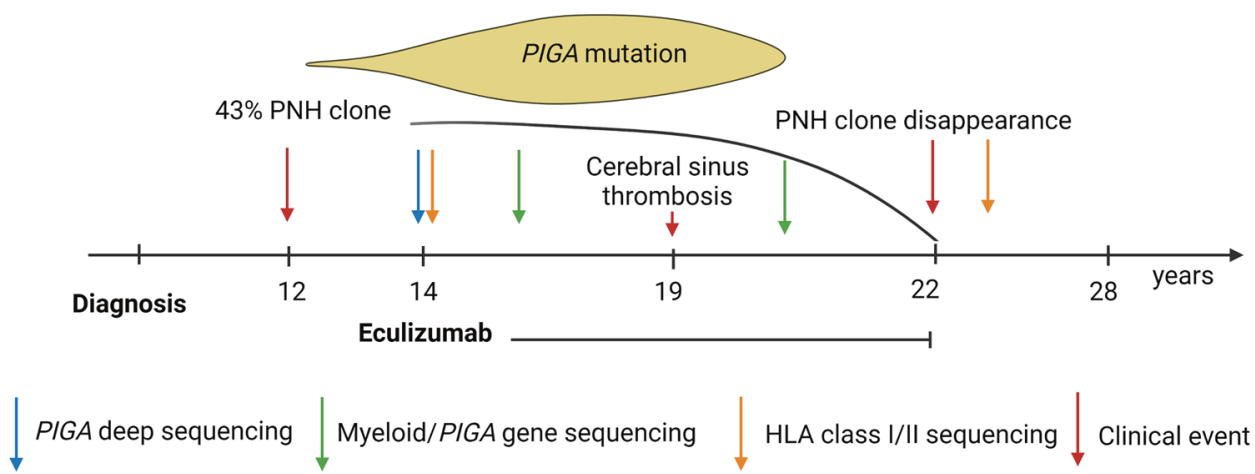

Fig. 1 Timeline of events for patients with PNH undergoing spontaneous remission. The figure showcases the disease course of the presented patients (UPN1-3) discussed in the main text as to clinical events (e.g., thrombotic complications, treatments), flow cytometric and molecular (PIGA, myeloid, HLA panels) data.

steroids and her course was complicated by a cerebral venous sinus thrombosis. Patient was eventually given eculizumab and her PNH clone started decreasing until it vanished (last 0.04\%) after 8 years from treatment start. Analysis of samples prior to and after PNH disappearance did not show any HLA class I/II nor myeloid driver gene mutations with absence of PIGA alterations at last sequencing. At last follow-up 6 years from eculizumab cessation, the patient persisted with normal blood counts.

Of note, in our patients with extinction of $\mathrm{PNH}$ cell populations anticomplement treatment cessation was never followed by recrudescence of the hemolytic process, nor there was evidence for recurrent AA.

PNH spontaneous remissions are rare events and still constitute an intriguing conundrum for the attending physicians. Through an extensive molecular study of longitudinal clonal dynamics, we demonstrated that in addition to be replaced by polyclonal (i.e., normal) hematopoiesis, PIGA clones may be swept by CHIP-like lesions in myeloid genes (e.g., $A S X L 1$ ), possibly characterized by improved fitness advantage in a process of Darwinian selection. In some cases, the retraction or even the exhaustion of a PNH clone may be the consequence of the restoration of normal hematopoiesis, as often observed in patients with AA receiving successful immunosuppressive treatment (as exemplified by UPN2) [17]. Alternatively, myeloid clones may represent the epiphenomenon of intricate dynamics of clonal succession, which affect a substantial proportion of PNH patients despite not driving any clear clinical outcome [10].
In summary, although the meaning of CHIP-like lesions in AA/ $\mathrm{PNH}$ remains to be fully elucidated, our results highlight the need for a watchful evaluation of clinically-apparent PNH remissions which, in some instances, can be replaced by conditions (e.g., CHIP) with a potential higher risk of malignant transformation. Future studies providing a magnified resolution of the molecular architecture of PNH clonal evolution at individual cell level (e.g., single-cell DNA sequencing) are warranted in order to potentially backtrack minor clones present prior to the disappearance of PNH clones, possibly presenting an increased fitness advantage in the "war of clones" of AA/PNH immunodynamics.

Carmelo Gurnari $\mathbb{D}^{1,2}$, Simona Pagliuca ${ }^{1,3}$, Tariq Kewan ${ }^{1}$, Waled Bahaj ${ }^{1}$, Minako Mori ${ }^{1}$, Bhumika J. Patel ${ }^{1}$,

Valeria Visconte (D) ${ }^{1}$ and Jaroslaw P. Maciejewski (D) ${ }^{1 \text { d́ }}$

${ }^{1}$ Department of Translational Hematology and Oncology Research, Taussig Cancer Institute, Cleveland Clinic, Cleveland, $\mathrm{OH}$, USA. ${ }^{2}$ Department of Biomedicine and Prevention, PhD in Immunology, Molecular Medicine and Applied Biotechnology, University of Rome Tor Vergata, Rome, Italy. ${ }^{3}$ University of Paris, Paris, France.

凶email: maciejj@ccf.org

DATA AVAILABILITY

Requests for additional information should be sent to the corresponding author. 


\section{REFERENCES}

1. Peffault de Latour R, Schrezenmeier $H$, Bacigalupo A, Blaise D, de Souza CA, Vigouroux $S$, et al. Allogeneic stem cell transplantation in paroxysmal nocturnal hemoglobinuria. Haematologica. 2012;97:1666-73.

2. Babushok DV, Stanley N, Xie HM, Huang H, Bagg A, Olson TS, et al. Clonal replacement underlies spontaneous remission in paroxysmal nocturnal haemoglobinuria. Br J Haematol. 2017;176:487-90.

3. Hillmen P, Lewis SM, Bessler M, Luzzatto L, Dacie JV. Natural history of paroxysmal nocturnal hemoglobinuria. N Engl J Med. 1995;333:1253-8.

4. Luzzatto L, Bessler M, Rotoli B. Somatic mutations in paroxysmal nocturnal hemoglobinuria: a blessing in disguise? Cell. 1997;88:1-4.

5. Korkama ES, Armstrong AE, Jarva $\mathrm{H}$, Meri S. Spontaneous remission in paroxysmal nocturnal hemoglobinuria-return to health or transition into malignancy? Front Immunol. 2018;9:1749.

6. Pulini S, Marando L, Natale A, Pascariello C, Catinella V, Del Vecchio L, et al. Paroxysmal nocturnal hemoglobinuria after autologous stem cell transplantation: extinction of the clone during treatment with eculizumab-pathophysiological implications of a unique clinical case. Acta Haematol. 2011;126:103-9.

7. Hakim F, Childs R, Balow J, Cowan K, Zujewski J, Gress R. Development of paroxysmal nocturnal hemoglobinuria after chemotherapy. Blood. 1996;88:4725-6.

8. Dingli D, Luzzatto L, Pacheco JM. Neutral evolution in paroxysmal nocturnal hemoglobinuria. Proc Natl Acad Sci USA. 2008;105:18496-500.

9. Mon Père N, Lenaerts T, Pacheco JM, Dingli D. Evolutionary dynamics of paroxysmal nocturnal hemoglobinuria. PLoS Comput Biol. 2018;14:e1006133.

10. Shen W, Clemente MJ, Hosono N, Yoshida K, Przychodzen B, Yoshizato T, et al. Deep sequencing reveals stepwise mutation acquisition in paroxysmal nocturnal hemoglobinuria. J Clin Invest. 2014;124:4529-38.

11. Yoshizato T, Dumitriu B, Hosokawa K, Makishima H, Yoshida K, Townsley D, et al. Somatic mutations and clonal hematopoiesis in aplastic anemia. N Engl J Med. 2015;373:35-47.

12. Gurnari C, Graham AC, Efanov A, Pagliuca S, Durrani J, Awada H, et al. Frequency and perturbations of various peripheral blood cell populations before and after eculizumab treatment in paroxysmal nocturnal hemoglobinuria. Blood Cells Mol Dis. 2020;87:102528.

13. Gurnari C, Pagliuca S, Patel BJ, Awada H, Kongkiatkamon S, Terkawi L, et al. Implication of PIGA genotype on erythrocytes phenotype in Paroxysmal Nocturnal Hemoglobinuria. Leukemia. 2021;35:2431-4. https://doi.org/10.1038/ s41375-020-01113-0.

14. Clemente MJ, Przychodzen B, Hirsch CM, Nagata Y, Bat T, Wlodarski MW, et al. Clonal PIGA mosaicism and dynamics in paroxysmal nocturnal hemoglobinuria. Leukemia. 2018;32:2507-11.

15. Hirsch CM, Nazha A, Kneen K, Abazeed ME, Meggendorfer M, Przychodzen BP, et al. Consequences of mutant TET2 on clonality and subclonal hierarchy. Leukemia. 2018;32:1751-61.

16. Nagata $Y$, Makishima H, Kerr CM, Przychodzen BP, Aly M, Goyal A, et al. Invariant patterns of clonal succession determine specific clinical features of myelodysplastic syndromes. Nat Commun. 2019;10:5386.

17. Scheinberg $P$, Marte $M$, Nunez $O$, Young NS. Paroxysmal nocturnal hemoglobinuria clones in severe aplastic anemia patients treated with horse anti-thymocyte globulin plus cyclosporine. Haematologica. 2010;95:1075-80.

\section{ACKNOWLEDGEMENTS}

We thank our sources of funding: the HENRY \& MARILYN TAUB FOUNDATION, grants R01HL118281, R01HL123904, R01HL132071, R35HL135795 (all to JPM), AA\&MDSIF (to W and JPM), VeloSano Pilot Award, Vera, and Joseph Dresner Foundation-MDS (to W), Foundation For Rare Diseases (FFRD) and Association HPN France-Aplasie medullaire (to SP). We thank Colin Hercus and Lucia D'Aprano for the bioinformatic support.

\section{AUTHOR CONTRIBUTIONS}

CG and JPM generated and conceived the study design, draw figures and tables, and wrote the manuscript; SP implemented the HLA mutational pipeline and analyzed molecular data; VV analyzed molecular data; SP, TK, WB, MM, and VV helped in data collection and analysis; BJP provided samples and edited the manuscript. All authors participated in the critical review of the final paper and submission.

\section{FUNDING INFORMATION}

GC was supported by the American-Italian Cancer Foundation Post-Doctoral Research Fellowship.

\section{COMPETING INTERESTS}

The authors declare no competing interests.

\section{ETHICS APPROVAL AND CONSENT TO PARTICIPATE}

Ethics approval and patients' consent to participate in the study were approved by The Institutional Review Boards of The Cleveland Clinic Foundation. All procedures were carried out in accordance with guidelines set forth by the Declaration of Helsinki.

\section{CONSENT FOR PUBLICATION}

Written informed consent was obtained from all patients.

\section{ADDITIONAL INFORMATION}

Supplementary information The online version contains supplementary material available at https://doi.org/10.1038/s41408-021-00582-5.

Correspondence and requests for materials should be addressed to Jaroslaw $\mathrm{P}$. Maciejewski.

Reprints and permission information is available at http://www.nature.com/ reprints

Publisher's note Springer Nature remains neutral with regard to jurisdictional claims in published maps and institutional affiliations.

\begin{abstract}
cc) (i) Open Access This article is licensed under a Creative Common Attribution 4.0 International License, which permits use, sharing, appropriate credit to the original author(s) and the source, provide a link to the Creative Commons license, and indicate if changes were made. The images or other third party material in this article are included in the article's Creative Commons license, unless indicated otherwise in a credit line to the material. If material is not included in the article's Creative Commons license and your intended use is not permitted by statutory regulation or exceeds the permitted use, you will need to obtain permission directly from the copyright holder. To view a copy of this license, visit http://creativecommons. org/licenses/by/4.0/.
\end{abstract}

(c) The Author(s) 2021 